Note

\title{
ALMOST SELF-COMPLEMENTARY UNIFORM HYPERGRAPHS ${ }^{1}$
}

\author{
Adam PaWee Wojda \\ AGH University of Science and Technology \\ Faculty of Applied Mathematics \\ Al. Mickiewicza 30,30-059 Kraków, Poland \\ e-mail: wojda@uci.agh.edu.pl
}

\begin{abstract}
A $k$-uniform hypergraph ( $k$-hypergraph) is almost self-complementary if it is isomorphic with its complement in the complete $k$-uniform hypergraph minus one edge. We prove that an almost self-complementary $k$-hypergraph of order $n$ exists if and only if $\left(\begin{array}{l}n \\ k\end{array}\right)$ is odd.
\end{abstract}

Keywords: uniform hypergraph.

2010 Mathematics Subject Classification: 035C. \footnotetext{
tion.

${ }^{1}$ The research was partially sponsored by the Polish Ministry of Science and Higher Educa-
} 


\section{REFERENCES}

[1] C.R.J. Clapham, Graphs self-complementary in $K_{n}-e$, Discrete Math. 81 (1990) 229-235. doi:10.1016/0012-365X(90)90062-M

[2] J.W.L. Glaisher, On the residue of a binomial coefficient with respect to a prime modulus, Quart. J. Math. 30 (1899) 150-156.

[3] L.N. Kamble, C.M. Deshpande and B.Y. Bam, Almost self-complementary 3-uniform hypergraphs, Discuss. Math. Graph Theory 37 (2017) 131-140. doi:10.7151/dmgt.1919

[4] S.H. Kimball, T.R. Hatcher, J.A. Riley and L. Moser, Solution to problem E1288 : Odd binomial coefficients, Amer. Math. Monthly 65 (1958) 368-369. doi: $10.2307 / 2308812$

[5] E.E. Kummer, Über die Ergänzungssätze zu den allgemeinen Reciprocitätsgesetzen, J. Reine Angew. Math. 44 (1852) 93-146. doi: $10.1515 /$ crll.1852.44.93

[6] E. Lucas, Sur les congruences des nombres eulériens st des coefficients differentiels, Bull. Soc. Math. France 6 (1878) 49-54. doi: $10.24033 /$ bsmf. 127

[7] G. Ringenboim, Fermat's Last Theorem for Amateurs (Springer Verlag, 1999).

[8] A. Szymański and A.P. Wojda, A note on $k$-uniform self-complementary hypergraphs of given order, Discuss. Math. Graph Theory 29 (2009) 199-202. doi:10.7151/dmgt.1440

[9] A.P. Wojda, Self-complementary hypergraphs, Discuss. Math. Graph Theory 26 (2006) 217-224. doi:/10.7151/dmgt.1314

Received 28 July 2017 Accepted 16 January 2017 